\title{
Mixed boundary node method for free vibration analysis of rectangular plates with variable thickness and general boundary conditions
}

\author{
M. Huang, J. Ma, J. N. Tang \& H. Yuan \\ School of Nuclear Technology and Engineering, \\ North China Electric Power University, P. R. China
}

\begin{abstract}
A mixed boundary node method is proposed for analyzing the free vibration of rectangular plates with variable thickness and general boundary conditions. The fundamental differential equations expressed by mixed variables are established and the unknown variables exist only on the partial boundary nodes. The relationship between the variables on the internal nodes and the pointed boundary nodes can be determined through using local regional integration and scanning technology. By utilizing boundary conditions, the discrete solutions for deflection of the plate with a concentrated load and arbitrary variable thickness are obtained and used to establish the eigen-value problem of matrix of the free vibration problem of plate. The convergence and accuracy of the numerical solutions for the natural frequency parameter calculated by the proposed method are investigated. The frequency parameters and their modes of free vibration are shown for some rectangular plates.

Key words: mixed variable, boundary node, free vibration, variable thickness, general boundary condition.
\end{abstract}

\section{Introduction}

Plates are common components in civil, aircraft and marine structures and have been extensively applied in practical engineering. Due to the material properties and the stress features of the plates, the internal force and 
deformation increase with the increasing of the diversity boundary conditions under loadings. Therefore, it is necessary to analyze the stress state of plates in order to provide some basis for plates design. The vibration analysis of plates is important for avoiding the resonance and has been studied for rectangular plates with thickness varying in one direction [1] and two directions [2].

Boundary element method (BEM) is an effectively numerical computational method used for solving linear partial differential equations to analyze the vibration problems of plates. It is to use the given boundary conditions to fit boundary values into the integral equations, rather than values throughout the space defined by partial differential equations. Applied in many fields of engineering and science, it has been developed alongside with finite element method (FEM) and finite difference method (FDM) providing more efficient such as reduction of the dimension of the system problem by one, high computational accuracy, less computing time and storage and well-suitable to an infinite domain problems. Tai and Shaw [3] first applied BEM to analyze the membrane vibration using a complex-valued kernel. Wong and Hutchinson [4] employed the direct BEM for solving clamped plates vibration. Xu Qiang [5] proposed an approach to free vibration analysis of plates based on the virtual boundary element method. The fundamental solution on the bending problem of the thin plate was applied to establish the integral equations on the virtual boundary of plate free vibration. The plate free vibration problem turned into algebraic eigenproblems which could be calculated directly. Wen [6] developed the dual boundary element equation to solve the cracked plate problem with dynamic loads by use of the moment integral equation. The applications of BEM to the dynamic characteristic of thin plate in lateral free vibration was presented by Fang Ying-wu [7].

Recent decades have witnessed numerous researches on the meshfree technique for numerical solution of partial differential equations (PDE) since the construction of meshes in the standard boundary element method is not benefit in dealing with moving boundary and complex geometric domain problems. Several meshless methods have been proposed such as smoothed particle hydrodynamics, element-free Galerkin, method of fundamental solution, boundary knot method and so on. Liu and Chen [8] used elementfree Galerkin to analyze the static deflection and the natural frequencies of thin plates of complicated shape. In their work, the moving least-squares (MLS) interpolation was used to construct shape functions through a set of nodes arbitrarily distributed in the domain. Tsai et al. [9] applied the method of fundamental solutions (MFS) to solve eigenfrequencies of plate vibration of multiply connected domains. The complex-valued MFS combined with the mix potential methods were utilized to avoid the spurious eigenvalues in their paper. Hon and Chen [10] extended the BKM to solve 2D Helmholtz equation and convection-diffusion problems for intricately irregular geometry

Based on the discrete method [11], a mixed boundary node method (MBNM) is proposed here to analyze the free vibration of rectangular plates 
with variable thickness and general boundary conditions. The method is a mesh-free method with unknown variables only on half of the boundary. Compared the discrete method [11], the present method can determine the relationship between the internal nodes and pointed boundary nodes rapidly by using local regional integration. Compared with other numerical computational methods, the present method provides some characteristics. Firstly, it does not require the fundamental solutions which are needed in the boundary element method, the method of fundamental solutions, the boundary knot method, etc. The starting point is to establish fundamental differential equations expressed by mixed variables. Secondly, the order of the differential equations is reduced to first-order from the forth-order by using mixed variables instead of deformation variables. That makes all the variables have the same accuracy. Thirdly, unknown variables exist only on the half of the boundary. That reduces the number of free degrees and the scale of the matrixes, therefore it can reduce calculating work and computing time. Lastly, the boundary conditions including torque conditions can be satisfied exactly on the pointed boundary nodes. In summary, the mixed boundary node method is an attractive numerical technique for less computational time, high accuracy and more efficient.

\section{Mixed boundary node method}

The fundamental differential equations of the plate with a concentrated load $\mathrm{P}$ at a point $\left(x_{q}, y_{r}\right)$, which are given by following equations.

$$
\begin{array}{ccc}
\frac{\partial Q_{x}}{\partial x}+\frac{\partial Q_{y}}{\partial y}+\bar{P} \delta\left(x-x_{q}\right) \delta\left(y-y_{r}\right)=0 & (1-\mathrm{a}) & \frac{\partial M_{x y}}{\partial x}+\frac{\partial M_{y}}{\partial y}-Q_{y}=0 \\
\frac{\partial M_{x}}{\partial x}+\frac{\partial M_{x y}}{\partial y}-Q_{x}=0 & (1-\mathrm{c}) & \frac{\partial \theta_{x}}{\partial x}+v \frac{\partial \theta_{y}}{\partial y}=\frac{M_{x}}{D} \\
\frac{\partial \theta_{y}}{\partial y}+v \frac{\partial \theta_{x}}{\partial x}=\frac{M_{y}}{D} & (1-\mathrm{e}) & \frac{\partial \theta_{x}}{\partial y}+\frac{\partial \theta_{y}}{\partial x}=\frac{2}{(1-v)} \frac{M_{x y}}{D} \\
\frac{\partial w}{\partial x}+\theta_{x}=\frac{Q_{x}}{G t_{s}} & (1-\mathrm{g}) & \frac{\partial w}{\partial y}+\theta_{y}=\frac{Q_{y}}{G t_{s}}
\end{array}
$$

where $Q_{x}, Q_{y}$ the shearing forces, $M_{x y}$ the twisting moment, $M_{x}, M_{y}$ the bending moments, $\theta_{x}, \theta_{y}$ the slopes, $w$ the deflection, $D=E h^{3} / 12\left(1-v_{2}\right)$ : the bending rigidity, $E, G$ : modulus, shear modulus of elasticity, $v$ :Poisson's ratio, $h=h(x, y)$ : the thickness of plate, $t_{s}=h / 1.2, \delta\left(x-x_{q}\right), \delta\left(x-x_{r}\right)$ : Dirac's delta functions.

By introducing the following non-dimensional expressions,

$$
\begin{gathered}
{\left[X_{1}, X_{2}\right]=\frac{a^{2}}{D_{0}\left(1-v^{2}\right)}\left[Q_{y}, Q_{x}\right],\left[X_{3}, X_{4}, X_{5}\right]=\frac{a}{D_{0}\left(1-v^{2}\right)}\left[M_{x y}, M_{y}, M_{x}\right],} \\
{\left[X_{6}, X_{7}, X_{8}\right]=\left[\theta_{y}, \theta_{x}, \omega / a\right] .}
\end{gathered}
$$

The differential equations $(1-\mathrm{a}) \sim(1-\mathrm{h})$ can be rewritten as follows

$$
\sum_{e=1}^{8}\left\{F_{1 t e} \frac{\partial X_{e}}{\partial \varsigma}+F_{2 t e} \frac{\partial X_{e}}{\partial \eta}+F_{3 t e} X_{e}\right\}+P \delta\left(\eta-\eta_{q}\right) \delta\left(\varsigma-\varsigma_{r}\right) \delta_{1 t}=0
$$

where $t=1 \sim 8, \mu=b / a, \eta=x / a, \zeta=y / b, D_{0}=E h_{0}^{3} / 12\left(1-v^{2}\right)$ : standard bending rigidity, $h_{0}$ : standard thickness of a plate. a,b: breadth, length of a 
rectangular plate, $P=\bar{P} a / D_{0}\left(1-v^{2}\right), \delta_{1 t}$ : Kronecker's delta, $F_{1 t e}, F_{2 t e}, F_{3 t e}$ : (Appendix I).

The plate can be considered as a group of discrete points shown in Fig. 1.

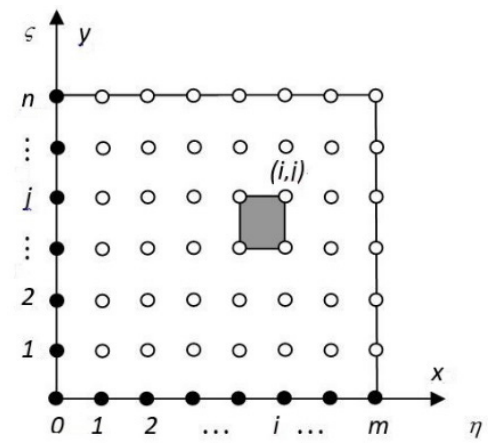

Figure 1: Discrete points on a rectangular plate.

In this paper, the specified point $(i, j)$ is chosen according to the regular order $(1,1),(1,2) \ldots(1, \mathrm{n}), \ldots(\mathrm{m}, 1),(m, 2) \ldots(m, n)$. By integrating equation $(2)$ over the area $[i, j]$ as shown in Fig. 1 , the following equation is obtained.

$$
\begin{aligned}
\sum_{e=1}^{8}\left\{F_{1 t e} \int_{\eta_{(i-1)}}^{\eta_{i}}[\right. & \left.X_{e}\left(\eta, \zeta_{j}\right)-X_{e}\left(\eta, \zeta_{(j-1)}\right)\right] d \eta+F_{2 t e} \int_{\zeta_{(j-1)}}^{\zeta_{j}}\left[X_{e}\left(\eta_{i}, \zeta\right)-X_{e}\left(\eta_{(i-1)}, \zeta\right)\right] d \zeta \\
& \left.+F_{3 t e} \int_{\eta_{(i-1)}}^{\eta_{i}} \int_{\zeta_{(j-1)}}^{\zeta_{j}} X_{e}(\eta, \zeta) d \eta d \zeta\right\}+P_{u}\left(\eta-\eta_{g}\right) u\left(\zeta-\zeta_{r}\right) \delta_{1 t}=0
\end{aligned}
$$

where $u\left(\eta, \eta_{q}\right), u\left(\zeta, \zeta_{i}\right)$ is the unit step function.

Next, by applying the numerical method, the simultaneous equation for the unknown quantities $X_{e i j}=X_{e}\left(\eta_{i}, \zeta_{j}\right)$ at point $(i, j)$ of the area $[i, j]$ is obtained as follows,

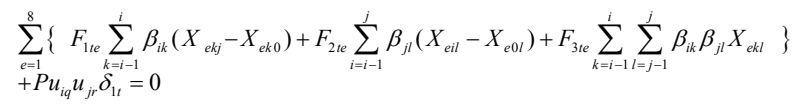

where $u_{i q}=0(i<q), u_{i q}=0.5(i=q), u_{i q}=1(i>q)$;

$$
u_{j r}=0(j<r), u_{j r}=0.5(j=r), u_{j r}=1(j>r) \text {. }
$$

The solution $X_{p i j}$ of the simultaneous equation (4) is obtained as follows,

$$
X_{p i j}=\sum_{d=1}^{6}\left\{\sum_{f=0}^{i} a_{p i j f d} X_{r f 0}+\sum_{g=0}^{j} b_{p i j g d} X_{r 0 g}\right\}+q_{p i j} P
$$

where

$$
\begin{aligned}
a_{p i j f d}=\sum_{t=1}^{8}\left\{\beta_{i i} A_{p t}\right. & {\left[a_{t(i-1)(j-1) f d}+a_{t i(j-1) f d}-a_{t(i-1) j f d}\right] } \\
& +\beta_{j j} B_{p t}\left[a_{t(i-1)(j-1) f d}+a_{t(i-1) j f d}-a_{t i(j-1) f d}\right] \\
& +\beta_{i i} \beta_{j j}\left[C_{p t(i-1)(j-1)} a_{t(i-1)(j-1) f d}+C_{p t(i-1) j} a_{t(i-1) j f d}\right. \\
& \left.\left.+C_{p t i(j-1)} a_{t i(j-1) f d}\right]\right\}
\end{aligned}
$$




$$
\begin{gathered}
b_{p i j g d}=\sum_{t=1}^{8}\left\{\beta_{i i} A_{p t}\left[b_{t(i-1)(j-1) g d}+b_{t i(j-1) g d}-b_{t(i-1) j g d}\right]\right. \\
+\beta_{j j} A B_{p t}\left[b_{t(i-1)(j-1) g d}+b_{t(i-1) j g d}-b_{t i(i-1) j g d}\right] \\
+\beta_{i i} \beta_{j j}\left[C_{p t(i-1)(j-1)} b_{t(i-1)(j-1) g d}+C_{p t(i-1) j} b_{t(i-1) j g d}\right. \\
\left.\left.+C_{p t(j-1)} b_{t i(j-1) g d}\right]\right\} \\
\bar{q}_{p i j}=\sum_{t=1}^{8}\left\{\beta_{i i} A_{p t}\left[\bar{q}_{t(i-1)(j-1)}+\bar{q}_{t i(j-1)}-\bar{q}_{t(i-1) j}\right]\right. \\
+\beta_{j j} B_{p t}\left[\bar{q}_{t(i-1)(j-1)}+\bar{q}_{t(i-1) j}-\bar{q}_{t i(j-1)}\right] \\
\left.+\beta_{i i} \beta_{j j}\left[C_{p t(i-1)(j-1)} \bar{q}_{t(i-1)(j-1)}+C_{p t(i-1) j} \bar{q}_{t(i-1) j}+C_{p t i(j-1)} q_{t i(j-1)}\right]\right\} \\
\quad-A_{p 1} u_{i p} u_{j r}
\end{gathered}
$$

$\beta_{i i}=\bar{h}_{i} / 2, \bar{h}_{i}=\eta_{i j}-\eta_{(i-1)}, \beta_{j j}=\bar{h}_{j} / 2, \bar{h}_{j}=\varepsilon_{i j}-\varepsilon_{i(j-1)}, p=1 \sim 8, A_{p t}, B_{p t}, C_{p t k}$ are given in Appendix II.

From the above expressions of the coefficients, it can be noted the two summations used in the method [11] have been changed to one summation. Therefore, the computing time of the coefficients can be reduced greatly. In this paper, three kinds of plate boundary conditions, namely, simply supported, fixed and cantilever are analyzed.

\section{Characteristic equation of free vibration}

By applying the Green function $\mathrm{w}\left(x_{0}, y_{0}, x, y\right) / \bar{P}$ which is the displacement at a point $\left(x_{0}, y_{0}\right)$ of a plate with a concentrated load $\bar{P}$ at a point $(x, y)$, the displacement amplitude $\widehat{\mathrm{w}}\left(x_{0}, y_{0}\right)$ at a point $\left(x_{0}, y_{0}\right)$ of the rectangular plate during the free vibration is given as follows.

$$
\widehat{w}\left(x_{0}, y_{0}\right)=\int_{0}^{b} \int_{0}^{a} \rho h \omega^{2} \widehat{w}(x, y)\left[w\left(x_{0}, y_{0}, x, y\right) / \bar{P}\right] d x d y
$$

where $\rho$ is the mass density of the plate material.

Choosing $\rho_{0}$ as the standard mass density and using the non-dimensional expressions,

$$
\begin{gathered}
\lambda^{4}=\frac{\rho_{0} h_{0} \omega^{2} a^{4}}{D_{0}\left(1-v^{2}\right)}, H(\eta, \zeta)=\frac{\rho(x, y) h(x, y)}{\rho_{0} h_{0}}, w(\eta, \zeta)=\frac{\widehat{w}(x, y)}{a}, \\
G\left(\eta_{0}, \zeta_{0}, \eta, \zeta\right)=\frac{w\left(x_{0}, y_{0}, x, y\right)}{a} \frac{D_{0}\left(1-v^{2}\right)}{\bar{P} a}, k=\frac{1}{\mu \lambda^{4}}
\end{gathered}
$$

the integral equation can be rewritten as follows:

$$
w\left(\eta_{0}, \zeta_{0}\right)=\int_{0}^{d} \int_{0}^{d} \mu \lambda^{4} H(\eta, \zeta) G\left(\eta_{0}, \zeta_{0}, \eta, \zeta\right) W(\eta, \zeta) d \eta d \zeta
$$


By applying the numerical integration method mentioned at the third-section, equation is discretely expressed as:

$$
k W_{k l}=\sum_{i=1}^{n+1} \sum_{j=1}^{n+1} H_{i j} G_{k l i j} W_{i j} k=\frac{1}{\mu^{4}}
$$

the homogeneous linear equations in $(m+l) \times(n+l)$ unknowns $W_{00}, W_{01}, W_{02}, \ldots$ $W_{0 \mathrm{n}}, W_{10}, W_{11}, W_{12}, \ldots W_{1 \mathrm{n}}, \ldots W_{\mathrm{m} 0}, W_{\mathrm{m} 1}, W_{\mathrm{m} 2}, \ldots W_{\mathrm{mn}}$ are obtained by using numerical integration as follows:

$$
\sum_{i=0}^{m} \sum_{j=0}^{n}\left(\beta_{m i} \beta_{n j} H_{i j} G_{k l i j}-\kappa \delta_{i k} \delta_{j l}\right) W_{i j}=0,(k=0,1, \ldots m, 1=0,1, \ldots n) .
$$

The characteristic equation of the free vibration of a rectangular plate with variable thickness is obtained from equation (8):

$$
\left|\begin{array}{ccccc}
K_{00} & K_{01} & K_{02} & \ldots & K_{0 m} \\
K_{10} & K_{11} & K_{12} & \ldots & K_{1 m} \\
K_{20} & K_{21} & K_{22} & \ldots & K_{2 m} \\
& \ldots & \ldots & \ldots & \\
K_{m 0} & K_{m 1} & K_{m 2} & \ldots & K_{m m}
\end{array}\right|=0
$$

where

$$
K_{i j}=\beta_{m j}\left[\begin{array}{cccc}
\beta_{n 1} H_{j 1} G_{i l j l}-k \delta_{i j} & \beta_{n 2} H_{j 2} G_{i l j 2} & \cdots & \beta_{n(n+l)} H_{j(n+l)} G_{i l j(n+l)} \\
\beta_{n 1} H_{j 1} G_{i 2 j 1} & \beta_{n 2} H_{j 2} G_{i 2 j 2}-k \delta_{i j} & \cdots & \beta_{n(n+l)} H_{j(n+l)} G_{i 2 j(n+1)} \\
\vdots & \vdots & \ddots & \vdots \\
\beta_{n 1} H_{j 1} G_{i(n+l) j 1} & \beta_{n 2} H_{j 2} G_{i(n+1) j 2} & \cdots & \beta_{n(n+1)} H_{j(n+l)} G_{i(n+l) j(n+1)}-k \delta_{i j}
\end{array}\right]
$$

\section{Numerical results}

The convergency and accuracy of numerical solutions have been investigated for the free vibration problem of some rectangular plates with variable thickness.

The convergent values of numerical solutions of frequency parameter for these plates have been obtained by using Richardson's extrapolation formula for two cases of combinations of divisional numbers $m$ and $n$.

\subsection{Simply supported rectangular plate with variable thickness}

Numerical solutions for the lowest twenty one natural frequency parameters $\lambda$ of a simply supported square plate and a rectangular plate of aspect ratio $b / a=2$ are shown in Table 1. The convergent values of numerical solutions were obtained by using Richardson's extrapolation formula for the two cases of division numbers $m(=n)$ of 12 and 16 for Ref. [12] by Leissa, and it shows the good convergency and satisfiable accuracy of the numerical solutions by present method. 
Table 1: $\quad$ Natural frequency parameter $\lambda$ for simple rectangular plate; $v=0.3$.

\begin{tabular}{|c|c|c|c|c|c|c|c|c|}
\hline \multirow{3}{*}{ mode } & \multicolumn{4}{|c|}{$\mathrm{b} / \mathrm{a}=1$} & \multicolumn{4}{|c|}{$\mathrm{b} / \mathrm{a}=2$} \\
\hline & \multicolumn{2}{|c|}{$\mathrm{m}$} & \multirow{2}{*}{$\begin{array}{c}\text { Extra- } \\
\text { polation }\end{array}$} & \multirow{2}{*}{ Ref.[4] } & \multicolumn{2}{|c|}{$\mathrm{m}$} & \multirow{2}{*}{$\begin{array}{c}\text { Extra- } \\
\text { polation }\end{array}$} & \multirow{2}{*}{ Ref.[4] } \\
\hline & 12 & 16 & & & 12 & 16 & & \\
\hline 1 & 4.574 & 4.563 & 4.548 & 4.549 & 3.617 & 3.607 & 3.596 & 3.596 \\
\hline 2 & 7.333 & 7.270 & 7.188 & 7.192 & 4.615 & 4.585 & 4.547 & 4.549 \\
\hline 3 & 7.333 & 7.270 & 7.188 & 7.192 & 6.029 & 5.924 & 5.789 & 5.799 \\
\hline 4 & 9.306 & 9.211 & 9.089 & 9.098 & 6.778 & 6.712 & 6.627 & 6.631 \\
\hline 5 & 10.672 & 10.442 & 10.146 & - & 7.793 & 7.511 & 7.148 & 7.192 \\
\hline 6 & 10.672 & 10.442 & 10.146 & - & 7.359 & 7.284 & 7.187 & 7.192 \\
\hline 7 & 12.110 & 11.873 & 11.569 & 11.597 & 8.318 & 8.192 & 8.030 & 8.041 \\
\hline 8 & 12.110 & 11.873 & 11.569 & 11.597 & 9.960 & 9.326 & 8.511 & 9.098 \\
\hline 9 & 14.530 & 13.931 & 13.161 & 13.262 & 9.672 & 9.403 & 9.056 & 8.661 \\
\hline 10 & 14.530 & 13.931 & 13.161 & 13.262 & 12.691 & 11.402 & 9.745 & 10.172 \\
\hline 11 & 14.374 & 14.037 & 13.603 & 13.647 & 10.299 & 10.062 & 9.757 & 9.782 \\
\hline 12 & 15.614 & 15.032 & 14.282 & - & 10.689 & 10.451 & 10.146 & 10.172 \\
\hline 13 & 15.614 & 15.032 & 14.282 & - & 11.489 & 10.906 & 10.156 & 10.298 \\
\hline 14 & 17.424 & 16.789 & 15.972 & 16.083 & 16.306 & 13.808 & 10.596 & - \\
\hline 15 & 17.424 & 16.789 & 15.972 & 16.083 & 11.369 & 11.102 & 10.757 & 10.789 \\
\hline 16 & 19.083 & 17.769 & 16.079 & - & 13.855 & 12.725 & 11.272 & - \\
\hline 17 & 19.083 & 17.769 & 16.079 & - & 12.393 & 12.022 & 11.545 & 11.597 \\
\hline 18 & 19.918 & 18.642 & 17.000 & 17.322 & 13.921 & 13.230 & 12.341 & - \\
\hline 19 & 19.918 & 18.642 & 17.000 & 17.322 & 14.259 & 13.650 & 12.865 & - \\
\hline 20 & 20.009 & 19.145 & 18.033 & 18.196 & 14.543 & 13.938 & 13.160 & - \\
\hline 21 & 21.360 & 20.081 & 18.436 & - & 15.049 & 14.432 & 13.639 & - \\
\hline
\end{tabular}

Table 2: $\quad$ Natural frequency parameter $\lambda$ for simple square plate with variable thickness; $v=0.3$.

\begin{tabular}{|c|c|c|c|c|c|c|c|c|}
\hline \multirow{3}{*}{ mode } & \multicolumn{4}{|c|}{$\alpha=0.1$} & \multicolumn{4}{|c|}{$\alpha=0.8$} \\
\hline & \multicolumn{2}{|c|}{$\mathrm{m}$} & \multirow{2}{*}{$\begin{array}{c}\text { Extra- } \\
\text { polation }\end{array}$} & \multirow{2}{*}{ Ref.[1] } & \multicolumn{2}{|c|}{$\mathrm{m}$} & \multirow{2}{*}{$\begin{array}{l}\text { Extra- } \\
\text { polatio }\end{array}$} & \multirow{2}{*}{ Ref.[1] } \\
\hline & 12 & 16 & & & 12 & 16 & & \\
\hline 1 & 4.687 & 4.675 & 4.660 & 4.661 & 5.386 & 5.372 & 5.354 & 5.335 \\
\hline 2 & 7.512 & 7.446 & 7.362 & - & 8.576 & 8.501 & 8.404 & - \\
\hline 3 & 7.513 & 7.447 & 7.363 & - & 8.611 & 8.535 & 8.437 & - \\
\hline 4 & 9.534 & 9.436 & 9.311 & - & 10.944 & 10.829 & 10.680 & - \\
\hline 5 & 10.927 & 10.692 & 10.389 & - & 12.342 & 12.080 & 11.742 & - \\
\hline 6 & 10.932 & 10.696 & 10.395 & - & 12.512 & 12.238 & 11.886 & - \\
\hline 7 & 12.405 & 12.162 & 11.850 & - & 14.210 & 13.926 & 13.560 & - \\
\hline 8 & 12.407 & 12.164 & 11.852 & - & 14.265 & 13.977 & 13.687 & - \\
\hline 9 & 14.870 & 14.269 & 13.496 & - & 16.581 & 15.918 & 15.066 & - \\
\hline 10 & 14.723 & 14.258 & 13.659 & - & 16.889 & 16.308 & 15.561 & - \\
\hline 11 & 14.883 & 14.378 & 13.730 & - & 17.018 & 16.481 & 15.791 & - \\
\hline 12 & 15.993 & 15.396 & 14.628 & - & 18.294 & 17.602 & 16.712 & - \\
\hline 13 & 15.997 & 15.400 & 14.631 & - & 18.402 & 17.701 & 16.799 & - \\
\hline \multirow{3}{*}{ mode } & \multicolumn{4}{|c|}{$\alpha=0.1$} & \multicolumn{4}{|c|}{$\alpha=0.8$} \\
\hline & \multicolumn{2}{|c|}{$\mathrm{m}$} & \multirow{2}{*}{$\begin{array}{l}\text { Extra- } \\
\text { polation }\end{array}$} & \multirow{2}{*}{ Ref.[1] } & \multicolumn{2}{|c|}{$\mathrm{m}$} & Extra- & \multirow{2}{*}{ Ref.[1] } \\
\hline & 12 & 16 & & & 12 & 16 & polatio & \\
\hline 14 & 17.846 & 17.195 & 16.451 & - & 21.467 & 20.047 & 18.222 & - \\
\hline 15 & 17.848 & 17.197 & 16.360 & - & 20.513 & 19.742 & 18.752 & - \\
\hline 16 & 19.514 & 18.174 & 16.451 & - & 20.428 & 19.670 & 18.695 & - \\
\hline 17 & 19.543 & 18.198 & 16.458 & - & 22.320 & 20.775 & 18.789 & - \\
\hline 18 & 20.397 & 19.091 & 17.411 & - & 23.299 & 21.798 & 19.867 & - \\
\hline 19 & 20.407 & 19.099 & 17.416 & - & 23.396 & 21.895 & 19.964 & - \\
\hline 20 & 20.482 & 19.607 & 18.469 & - & 23.481 & 22.446 & 21.116 & - \\
\hline 21 & 21.874 & 20.564 & 18.880 & - & 23.992 & 23.484 & 21.546 & - \\
\hline
\end{tabular}


Table 3: $\quad$ Natural frequency parameter $\lambda$ for simple rectangular plate with variable thickness; $v=0.3$.

\begin{tabular}{|c|c|c|c|c|c|c|c|c|}
\hline \multirow{3}{*}{ mode } & \multicolumn{4}{|c|}{$\alpha=1$} & \multicolumn{4}{|c|}{$\alpha=2$} \\
\hline & \multicolumn{2}{|c|}{$\mathrm{m}$} & \multirow{2}{*}{$\begin{array}{c}\text { Extra- } \\
\text { polation }\end{array}$} & \multirow{2}{*}{ Ref.[4] } & \multicolumn{2}{|c|}{$\mathrm{m}$} & \multirow{2}{*}{$\begin{array}{c}\text { Extra- } \\
\text { polation }\end{array}$} & \multirow{2}{*}{ Ref.[4] } \\
\hline & 12 & 16 & & & 12 & 16 & & \\
\hline 1 & 3.705 & 3.696 & 3.684 & 3.684 & 4.2446 & 4.234 & 4.220 & 4.221 \\
\hline 2 & 4.728 & 4.698 & 4.659 & - & 5.433 & 5.398 & 5.352 & - \\
\hline 3 & 6.176 & 6.069 & 5.930 & - & 7.078 & 6.955 & 6.797 & - \\
\hline 4 & 6.943 & 6.876 & 6.789 & - & 7.955 & 7.876 & 7.775 & - \\
\hline 5 & 7.982 & 7.693 & 7.322 & - & 9.101 & 8.776 & 8.359 & - \\
\hline 6 & 7.539 & 7.462 & 7.362 & - & 8.641 & 8.552 & 8.436 & - \\
\hline 7 & 8.521 & 8.392 & 8.226 & - & 9.775 & 9.624 & 9.430 & - \\
\hline 8 & 9.909 & 9.633 & 8.903 & - & 11.547 & 10.831 & 9.910 & - \\
\hline 9 & 10.200 & 9.551 & 9.091 & - & 11.377 & 11.055 & 10.641 & - \\
\hline 10 & 10.550 & 10.307 & 9.995 & - & 14.574 & 13.145 & 11.308 & - \\
\hline 11 & 12.992 & 11.673 & 9.977 & - & 12.072 & 11.791 & 11.430 & - \\
\hline 12 & 10.949 & 10.706 & 10.393 & - & 12.533 & 12.250 & 11.886 & - \\
\hline 13 & 11.770 & 11.173 & 10.405 & - & 13.529 & 12.833 & 11.938 & - \\
\hline 14 & 16.682 & 14.132 & 10.853 & - & 18.498 & 15.784 & 12.295 & - \\
\hline 15 & 11.647 & 11.373 & 11.021 & - & 13.336 & 13.017 & 12.813 & - \\
\hline 16 & 14.262 & 13.036 & 11.460 & - & 16.47 & 14.984 & 13.154 & - \\
\hline 17 & 12.695 & 112.315 & 11.826 & - & 14.545 & 14.102 & 13.532 & - \\
\hline 18 & 14.262 & 13.552 & 12.728 & - & 16.275 & 15.527 & 14.565 & - \\
\hline 19 & 14.605 & 13.980 & 13.177 & - & 16.698 & 15.977 & 15.050 & - \\
\hline 20 & 14.896 & 14.276 & 13.479 & - & 17.033 & 16.317 & 15.396 & - \\
\hline 21 & 15.414 & 14.781 & 13.967 & - & 17.628 & 16.897 & 15.957 & - \\
\hline
\end{tabular}

Numerical solutions for the lowest twenty one natural frequency parameters $\lambda$ of a simply supported square plate and a rectangular plate of aspect ratio $b / a=2$ with a linear thickness variation in the $\eta$-direction given by $h(\eta, \zeta)=h_{0}(1+\alpha \eta)$ are shown in Table 2 and 3 for two cases of $\alpha=0.1$ and 0.8 . The convergent values of numerical solution were obtained for the two cases of divisional numbers $m(=n)$ of 12 and 16 for the whole part of the plate. Table 2 and 3 involves the other theoretical values of the fundamental frequency by Apple and Byers [1]. The numerical solutions by present method have the good convergency and satisfiable accuracy of fundamental frequency.

\subsection{Fixed rectangular plate with variable thickness}

Numerical solutions for the lowest twenty one natural frequency parameters $\lambda$ of a fixed square plate and a rectangular plate of aspect ratio $b / a=2$ are obtained for the two cases of divisional numbers $m(=n)$ of 12 and 16 for the whole part of the plate. Table 4 involves the other theoretical values by Claassen and Thorne [13]. The numerical solutions by the present method have the good convergency and satisfiable accuracy.

Numerical solutions for the lowest twenty one natural frequency parameters $\lambda$ of a fixed square with a sinusoidal thickness variation in the $\eta$, $\zeta$-directons given by $h(\eta, \zeta)=h_{0}(1-\alpha \sin \pi \eta)(1-\alpha \sin \pi \zeta)$ are shown in Table 5 for two cases of $\alpha=0.3$ and 0.5 . The convergent values of numerical solutions were obtained for the two cases of divisional numbers $m(=n)$ of 12 and 16 for the whole part of the plate. 
Table 4: $\quad$ Natural frequency parameter $\lambda$ for fixed rectangular plate; $v=0.3$.

\begin{tabular}{|c|c|c|c|c|c|c|c|c|}
\hline \multirow{3}{*}{ mode } & \multicolumn{4}{|c|}{$\mathrm{b} / \mathrm{a}=1$} & \multicolumn{4}{|c|}{$\mathrm{b} / \mathrm{a}=2$} \\
\hline & \multicolumn{2}{|c|}{$\mathrm{m}$} & \multirow{2}{*}{$\begin{array}{c}\text { Extra- } \\
\text { polation }\end{array}$} & \multirow{2}{*}{ Ref.[5] } & \multicolumn{2}{|c|}{$\mathrm{m}$} & \multirow{2}{*}{$\begin{array}{c}\text { Extra- } \\
\text { polation }\end{array}$} & \multirow{2}{*}{ Ref.[5] } \\
\hline & 12 & 16 & & & 12 & 16 & & \\
\hline 1 & 6.205 & 6.175 & 6.138 & 6.142 & 5.133 & 5.107 & 5.073 & 5.076 \\
\hline 2 & 9.030 & 8.911 & 8.756 & 8.771 & 5.883 & 5.834 & 5.771 & 5.776 \\
\hline 3 & 9.030 & 8.911 & 8.756 & 8.771 & 7.175 & 7.023 & 6.829 & 6.851 \\
\hline 4 & 10.985 & 10.829 & 10.629 & 10.651 & 8.970 & 8.573 & 8.064 & 8.148 \\
\hline 5 & 12.533 & 12.162 & 11.686 & 11.745 & 8.475 & 8.344 & 8.176 & 8.19 \\
\hline 6 & 12.563 & 12.191 & 11.714 & 11.772 & 8.923 & 8.798 & 8.617 & 8.632 \\
\hline 7 & 13.910 & 13.551 & 13.091 & 13.152 & 11.305 & 10.430 & 9.305 & 9.668 \\
\hline 8 & 13.910 & 13.551 & 13.091 & 13.152 & 9.742 & 9.558 & 9.320 & 9.343 \\
\hline 9 & 16.690 & 15.803 & 14.663 & 14.856 & 11.023 & 10.667 & 10.209 & 10.279 \\
\hline 10 & 16.690 & 15.803 & 14.663 & 14.856 & 14.372 & 12.611 & 10.347 & - \\
\hline 11 & 16.194 & 15.717 & 15.105 & - & 16.671 & 14.087 & 10.765 & - \\
\hline 12 & 17.655 & 16.817 & 15.741 & 15.933 & 18.600 & 15.193 & 10.813 & 11.044 \\
\hline 13 & 17.697 & 16.856 & 15.744 & - & 12.892 & 12.134 & 11.160 & - \\
\hline 14 & 19.420 & 18.550 & 17.432 & - & 12.196 & 11.808 & 11.309 & - \\
\hline 15 & 19.421 & 18.550 & 17.432 & - & 12.505 & 12.123 & 11.632 & - \\
\hline 16 & 21.676 & 19.825 & 17.446 & - & 15.565 & 13.991 & 11.970 & - \\
\hline 17 & 21.684 & 19.833 & 17.454 & - & 13.074 & 12.672 & 12.155 & - \\
\hline 18 & 22.409 & 20.633 & 18.351 & - & 13.995 & 13.492 & 12.845 & - \\
\hline 19 & 22.409 & 20.633 & 18.351 & - & 15.422 & 14.626 & 13.603 & - \\
\hline 20 & 22.063 & 20.929 & 19.471 & 19.712 & 16.444 & 15.536 & 14.369 & - \\
\hline 21 & 23.707 & 21.982 & 19.765 & - & 17.610 & 16.193 & 15.466 & - \\
\hline
\end{tabular}

Table 5: $\quad$ Natural frequency parameter $\lambda$ for fixed square plate with variable thickness; $v=0.3$.

\begin{tabular}{|c|c|c|c|c|c|c|}
\hline \multirow{3}{*}{ mode } & \multicolumn{3}{|c|}{$\alpha=0.3$} & \multicolumn{3}{|c|}{$\alpha=0.5$} \\
\hline & \multicolumn{2}{|c|}{$\mathrm{m}$} & \multirow{2}{*}{ Extra-polation } & \multicolumn{2}{|c|}{$\mathrm{m}$} & \multirow{2}{*}{ Extra-polation } \\
\hline & 12 & 16 & & 12 & 16 & \\
\hline 1 & 5.097 & 5.071 & 5.038 & 4.315 & 4.292 & 4.262 \\
\hline 2 & 7.225 & 7.128 & 7.003 & 5.944 & 5.863 & 5.758 \\
\hline 3 & 7.225 & 7.128 & 7.003 & 5.944 & 5.863 & 5.758 \\
\hline 4 & 8.866 & 8.736 & 8.570 & 7.360 & 7.243 & 7.093 \\
\hline 5 & 9.858 & 9.563 & 9.185 & 7.965 & 7.724 & 7.415 \\
\hline 6 & 9.894 & 9.599 & 9.220 & 7.965 & 7.726 & 7.420 \\
\hline 7 & 11.172 & 10.878 & 10.500 & 9.218 & 8.961 & 8.631 \\
\hline 8 & 11.172 & 10.878 & 10.500 & 9.218 & 8.961 & 8.631 \\
\hline 9 & 13.027 & 12.329 & 11.431 & 10.381 & 9.824 & 9.108 \\
\hline 10 & 13.027 & 12.329 & 11.431 & 10.381 & 9.824 & 9.108 \\
\hline 11 & 13.033 & 12.640 & 12.135 & 10.811 & 10.459 & 10.006 \\
\hline 12 & 14.119 & 13.438 & 12.561 & 11.560 & 10.986 & 10.248 \\
\hline 13 & 14.167 & 13.481 & 12.599 & 11.601 & 11.023 & 10.280 \\
\hline 14 & 16.844 & 15.393 & 13.528 & 13.318 & 12.174 & 10.703 \\
\hline 15 & 16.846 & 15.397 & 13.534 & 13.315 & 12.174 & 10.707 \\
\hline 16 & 15.630 & 14.913 & 13.990 & 12.961 & 12.327 & 11.512 \\
\hline 17 & 15.630 & 14.913 & 13.990 & 12.961 & 12.327 & 11.512 \\
\hline 18 & 17.885 & 16.447 & 14.599 & 14.535 & 13.357 & 11.842 \\
\hline 19 & 17.885 & 16.447 & 14.599 & 14.535 & 13.357 & 11.842 \\
\hline 20 & 17.885 & 16.842 & 15.642 & 14.795 & 13.962 & 12.890 \\
\hline 21 & 190.95 & 17.670 & 15.837 & 15.780 & 14.545 & 12.957 \\
\hline
\end{tabular}

\section{Conclusions}

A mixed boundary node method was proposed for analyzing the free vibration problem of various types of rectangular plates with uniform or nonuniform 
thickness. Due to numerical works, it was shown that the numerical solutions by the proposed method had the good convergency and satisfiable accuracy for various type of rectangular plates with uniform or non-uniform thickness.

\title{
Appendix I
}

$\mathrm{F}_{111}-\mathrm{F}_{123}-\mathrm{F}_{134}-\mathrm{F}_{146}-\mathrm{F}_{167}-\mathrm{F}_{178}-\mathrm{F}_{188}-1, \mathrm{~F}_{212}-\mathrm{F}_{225}-\mathrm{F}_{233}-\mathrm{F}_{257}-\mathrm{F}_{266}-\mu, \mathrm{F}_{156}-\nu \mu, \mathrm{F}_{322}=\mathrm{F}_{331}=-\mu$, $\mathrm{F}_{344}=\mathrm{F}_{355}=-\mathrm{I}, \mathrm{F}_{363}=-\mathrm{J}, \mathrm{F}_{372}=-\mathrm{k}, \mathrm{F}_{377}=1, \mathrm{~F}_{381}=-\mu \mathrm{k}, \mathrm{F}_{386}=\mu$, other $\quad \mathrm{F}_{1 \mathrm{e}}=\mathrm{F}_{2 \mathrm{te}}=\mathrm{F}_{3 \mathrm{te}}=0$, $\mathrm{I}=\mu\left(1-\mathrm{v}^{2}\right)\left(\mathrm{h}_{0} / \mathrm{h}\right)^{3}, \mathrm{k}=(1 / 10)(\mathrm{E} / \mathrm{G})\left(\mathrm{h}_{0} / \mathrm{h}\right)^{2}\left(\mathrm{~h}_{0} / \mathrm{h}\right)$

\section{Appendix II}

\begin{abstract}
$\mathrm{A}_{p 1}=\Upsilon_{p 1}, \mathrm{~A}_{p 2}=0, \mathrm{~A}_{p 3}=\Upsilon_{p 2}, \mathrm{~A}_{p 4}=\Upsilon_{p 3}, \mathrm{~A}_{p 5}=0, \mathrm{~A}_{p 6}=\Upsilon_{p 4}+v \Upsilon_{p 5}, \mathrm{~A}_{p 7}=\Upsilon_{p 6}, \mathrm{~A}_{p 8}=\Upsilon_{p 7}$, $\mathrm{B}_{p 1}=0, \mathrm{~B}_{p 2}=\mu \Upsilon_{p 1}, \mathrm{~B}_{\mathrm{p} 3}=\mu \Upsilon_{p 3}, \mathrm{~B}_{p 4}=0, \mathrm{~B}_{p 5}=\mu \Upsilon_{p 2}, \mathrm{~B}_{p 6}=\mu \Upsilon_{p 6}, \mathrm{~B}_{p 7}=\mu\left(v \Upsilon_{p 1}+v \Upsilon_{p 5}\right)$, $\mathrm{B}_{p 8}=v \Upsilon_{p 8}, \quad \mathrm{C}_{p l k l}=\mu \Upsilon_{p 3}+k_{k l} \Upsilon_{p 7}, \quad \mathrm{C}_{p 2 k l}=\mu \Upsilon_{p 2}+k_{k l} \Upsilon_{p 8}, \quad \mathrm{C}_{p 3 k l}=J \Upsilon_{p 6}, \quad \mathrm{C}_{p 4 k l}=I_{k l} \Upsilon_{p 4}$, $\mathrm{C}_{p 5 k l}=I_{k l} \Upsilon_{p 5}, \mathrm{C}_{p 6 k l}=-\mu \Upsilon_{p 7}, \mathrm{C}_{p 7 k l}=-\Upsilon_{p 8}, \mathrm{C}_{p 8 k l}=0,\left[\Upsilon_{p k}\right]=\left[\bar{\Upsilon}_{p k}\right]^{-1},\left[\bar{\Upsilon}_{11}\right]=\beta_{i i}, \bar{\Upsilon}_{12=} \mu \beta_{j j}$,

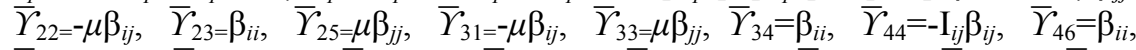

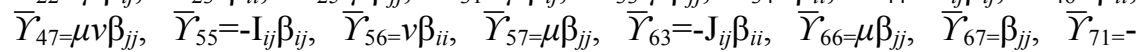
$\mu k_{i j} \beta_{j j}, \bar{Y}_{76=\mu \beta_{i j}}, \bar{Y}_{78=} \beta_{i i}, \bar{Y}_{82=-k_{i j}} \beta_{i j}, \bar{Y}_{87=} \beta_{i j}, \bar{Y}_{88=} \beta_{j j}$, other $\bar{Y}_{p k=} 0, \beta_{i j}=\beta_{i i} \beta_{j j}$
\end{abstract}

\section{References}

[1] F. C. Apple and N. R. Byers, Fundamental Frequency of Simply Sup-ported Rectangular Plates with Linearly Varying Thickness. J. Appl. Mech: 32, No.1, 163-167, 1965.

[2] R. Plunkett, Natural Frequencies of Uniform and Non-Uniform Rectangular Cantilever Plates, J. Mech. Eng. Sci.,5, No.2, 146-156, 1963.

[3] Tai G.R.G., Shaw R.P., Helmholtz equation eigenvalues and eigenmodes for arbitrary domains. Journal of Acoustical Society of America: 56:796 804, 1974.

[4] Hutchinson J. R., Wong G. K. K., The boundary element method for plate vibrations. Proceedings of the ASCE 7th Conference on Electronic Computation, St. Louis, Missouri. ASCE: New York, 297-311, 1979.

[5] Xu Qiang, Zhu Jin-long, Zhao Zhi, New approach to free vibration analysis of plate using virtual boundary element. Journal of Tongji University.

[6] P. H. Wen, M. H. Aliabadi, A. Young, A boundary element method for dynamic plate bending problems. International Journal of Solids and Structures 37, pp. 5177-5188, 2000.

[7] Fang Ying-wu, Huang Yu-mei, Zhang Guang-peng, Wei Jun-chao, Analysis for Free Vibration of Thin Plate by Boundary Element Method. Journal of Xi'an University of Technology, Vol. 20 No. 2, 2004. 
[8] G. R. Liu and X. L. Chen, A Mesh-Free Method for Static and Free Vibration Analyses of Thin Plates of Complicated Shape. Journal of Sound and Vibration: 241(5), 839-855, 2001.

[9] C. C. Tsai, D. L. Young and C. M. Fan, Method of Fundamental Solutions for Plate Vibrations in Multiply Connected Domains. Journal of Mechanics: Vol. 22, pp. 235-245, 2006.

[10] W. Chen and Y. C. Hon. Numerical investigation on convergence of boundary knot method in the analysis of homogeneous Helmholtz, modified Helmholtz, and convection-diffusion problems. Computer methods in applied mechanics and engineering, Vol. 192, 1859-1875, 2003.

[11] M. Huang, X. Q. Ma, H. Matuda and C. Morita Free vibration analysis of continuous rectangular plates. Journal of Sound and Vibration 2010, 329: 485-496.

[12] A. W. Leissa: Vibration of Plates, NASA SP-160, 43-45, 1969.

[13] R. W. Claassen and C. J. Thorne, Transverse Vibrations of Thin Rectangular Isotropic Plates. NOTS Tech. Pub: 2379, NAVWEPS Rept. 7016, U.S. Naval Ordnance Test Sta, 1960.

[14] R. W. Claassen and C. J. Thorne: Vibrations of a Rectangular Cantierer Plate. J. Aerospace Sci., 29, No.11, 1300-1305, 1962. 